cirrhosis will undergo endoscopic variceal surveillance every 1-3 years depending on the presence of varices.

OGD is a key diagnostic test with one of the longest waiting lists in the NHS and variceal surveillance contributes to the overall waits in upper GI endoscopy. It is therefore essential that the appropriate patients are captured in order to maximise both the efficacy of the service and also the financial cost of delivering the service.

The Baveno VI criterion uses biochemical and non-invasive measurements to predict the presence of oesophageal varices in patients with compensated cirrhosis. Based on the criteria, a platelet count $>150 \times 109 / \mathrm{L}$ and a Liver Stiffness Measurement (LSM) $<20 \mathrm{kPa}$ are deemed at low risk of oesophageal varices and could avoid an OGD for variceal surveillance.

We proposed to apply the Baveno VI criteria in our regional liver unit to identify patients that may be able to avoid having an OGD for variceal surveillance.

Methodology A retrospective analysis was completed utilising the database of cirrhotic patients registered at Aintree University Hospital, Liverpool. Patients that met Baveno VI criteria and had an OGD were included.

OGD reports from 6 July 2001 - 16 February 2021 were obtained from the endoscopy database (UniSoft). Fibroscan reports were available for patients from 26 August 2015 - 16 February 2021.

Results 623 patients were registered on the cirrhosis database of which $62(10 \%)$ fulfilled inclusion criteria. Alcohol and NASH were the predominant aetiologies $(24 \%$ and $43.5 \%$ respectively). Median age at time of Fibroscan was 60 years (30-80), median LSM was $13 \mathrm{kPa}(3.2-19.8)$, median platelet count 219x109/L (157x109/L-432x109/L). $3.2 \%$ ( 2 out of 62 ) patients had oesophageal varices (1 with Grade 1 and 1 with Grade 2 varices). 30 patients had an OGD within a year of their Fibroscan. The positive predictive value was $96.8 \%$. Varices requiring treatment in this cohort was $1.6 \%$.

Conclusion The application of Baveno VI criteria can safely and accurately decrease the number of OGD's required within a variceal surveillance program, reducing the risk of invasive procedures and resulting in a cost saving and reduction on pressures on waiting lists for OGD.

\section{P083 IMPACT OF COVID INFECTION ON OUTCOME OF PATIENTS WITH ACUTE ON CHRONIC LIVER FAILURE}

Shemin Kovammal ${ }^{*}$, Jayshri Shah, Glenda Osuoha, Seema Zulfikar, Syed Asfandyar. William Harvey Hospital, EKHUFT, Ashford, UK, Introduction

\subsection{6/gutjnl-2021-BASL.91}

Acute on chronic liver failure (ACLF) is a syndrome occurring in patients with chronic liver disease with or without cirrhosis characterised by acute hepatic decompensation and one or more extra hepatic organ failure with an increased mortality. Numerous factors have been studied which influence the outcome of these patients.

Aim To assess the impact of COVID infection on management and treatment escalation of patients who presented with acute hepatic decompensation with known or unknown underlying chronic liver disease after risk stratification.

Methodology Retrospective analysis of all patients admitted over two periods - Pre Covid (August 2019- March 2020-
Group A) and Covid (April 2020 - December 2020- Group B) with acute decompensated liver disease at our hospital. Data was analyzed from patient notes and electronic records on the following variables: age, gender, etiology of liver disease, prognostication scores - Child Pugh, MELD, ACLF grade and WHO performance status. CLIF -C score was calculated for patients referred to intensive care unit. Patient outcome measures - mortality and duration of hospital stay was also recorded. Data was collected in $35 / 72$ patients who were admitted in Group A and 31/95 patients in Group B due to non-availability of all patient notes.

Results 6/35 patients (17\%) died in Group A compared to 13/ 31(41.9\%) patients in Group B. 8/31 had COVID pneumonitis, 7 died and 1 survived. Alcohol was the commonest cause of underlying liver disease in both groups (Group A 32/35, Group B 27/31). Decompensation episodes in Group A versus Group B are as follows: gastrointestinal bleed 17/35 (48\%), 16/31(51\%); Hepatic encephalopathy 9/35(25\%), 6/31(19\%); Spontaneous Bacterial peritonitis 9/35(25\%), 9/31(29\%) respectively. Table 1 shows median MELD, Childs Pugh, ACLF grade was almost similar in both groups along with further

\begin{tabular}{|c|c|c|}
\hline & Pre Covid/Group A $(n=35)$ & Covid/Group B $(n=31)$ \\
\hline Mean Age & $56.63 \pm 12.5 \mathrm{SD}$ & $59.16 \pm 10.75$ SD \\
\hline \multirow[t]{2}{*}{ Gender } & $21(60 \%)$ & $20(64.5 \%)$ \\
\hline & $14(40 \%)$ & $11(35.5 \%)$ \\
\hline Median MELD Score & 21 (Range 6-40) & 21(Range 9-32) \\
\hline Median Child Pugh Score & 11 (Rang 6-14) & 12(Range 10-15) \\
\hline Median ACLF Score & 8 (Range 6-11) & 8 (Range $6-15$ ) \\
\hline No ACLF & $27(77 \%)$ & $21(67 \%)$ \\
\hline ACLF grade 1 & 5 & 4 \\
\hline ACLF grade 2 & 2 & 5 \\
\hline ACLF grade3 & 1 & 1 \\
\hline Average Performance score & 1 (Range $0-2$ ) & 1 (Range $0-3$ ) \\
\hline \multicolumn{3}{|l|}{ Patients referred to ITU } \\
\hline Patients referred to ITU & $4(11 \%)$ & $8(25 \%)$ \\
\hline Patients accepted for ITU & 3 & 6 \\
\hline Median CLIF - C Score & 54 (Range 45-58) & 56 (Range 51- 61) \\
\hline Average Performance Score & 1 (Range $0-1$ ) & 1 (Range $0-1$ ) \\
\hline Average ITU days & 9 (Range $6-13$ ) & 7 (Range 1-16) \\
\hline Mortality & $1(25 \%)$ & $4(66.6 \%)$ \\
\hline \multicolumn{3}{|c|}{ Patients who received ward-based care } \\
\hline $\begin{array}{l}\text { Number of patients admitted for } \\
\text { ward-based care }\end{array}$ & $32(91.4 \%)$ & $25(80.6 \%)$ \\
\hline Median MELD score & 18 (Range 6-27) & 21 (Range (9-32) \\
\hline Average hospital days & 14 days (Range $3-80$ ) & 16 days (Range $3-31$ ) \\
\hline Mortality & $5(16 \%)$ & $9(36 \%)$ \\
\hline $\begin{array}{l}\text { Median MELD score for patients } \\
\text { with mortality }\end{array}$ & 21 (Range 21- 28) & 26 (Range 13- 40) \\
\hline \multicolumn{3}{|c|}{ Patients who recovered \& got Discharged } \\
\hline Number of patients & $29(82.8 \%)$ & $18(58 \%)$ \\
\hline Median MELD score & 15 (Range 6-22) & 19 (Range 9 - 28) \\
\hline Median ACLF score & 7(Range 6-11) & 8(Range 6- 11) \\
\hline Average Performance Score & 1 (Range 0 - 2) & 1 (Range $0-2$ ) \\
\hline \multicolumn{3}{|l|}{ Patients who Died } \\
\hline Number of patients & $6(17 \%)$ & $13(41 \%)$ \\
\hline Median MELD Score & 21(Range $18-28$ ) & 28 (Range 13-40) \\
\hline Median ACLF score & 8 (Range 6-11) & 9 (Range $6-15$ ) \\
\hline Average Performance Score & 1 (Range $0-2$ ) & 1 (Range $0-2$ ) \\
\hline
\end{tabular}


analysis into patients who recovered or died depending on ward based care versus ITU.

Conclusion COVID pneumonitis influenced the outcome of patients admitted with hepatic decompensation resulting in increased mortality. Alcohol was the commonest cause of underlying liver disease in both Pre-COVID and COVID period. Age, nature of hepatic decompensation (Gastrointestinal bleeding, hepatic encephalopathy, spontaneous bacterial peritonitis) CLIF-C score for patients referred to ITU and average hospital stay was not much different in both the groups. The median MELD and ACLF grade were higher in patients who died compared to those who recovered.

\section{P084 A MELD SCORE $\geq 19$ PRIOR TO ANTI-THYMOCYTE GLOBULIN THERAPY FOR STEROID RESISTANT T CELL- MEDIATED REJECTION PREDICTS POOR 5-YEAR TRANSPLANT FREE SURVIVAL}

Lindsay Smith*, Oliver Tavabie, Niloufar Safinia, Cathrine McKenzie, Andreas Prachalias, Krishna Menon, Kosh Agarwal, Michael Heneghan, Varuna Aluvihare. King's College Hospital, UK

\subsection{6/gutjnl-2021-BASL.92}

Background and Aims Steroid resistant $\mathrm{T}$ cell-mediated rejection is a risk factor for graft loss and patient mortality post liver transplant (LT). Anti-thymocyte globulin (ATG) therapy has been demonstrated to be an effective treatment for this condition but long term follow up data are limited. We aimed to identify risk factors for reduced 1-year and 5-year LT free survival post ATG use for steroid resistant $\mathrm{T}$ cell-mediated rejection.

Method We performed a retrospective observational study evaluating all LT patients who received ATG for steroid resistant cellular rejection between 01/2007-01/2020 at King's College Hospital. ATG was administered based on institutional practice and clinician choice. Patients were excluded if they received ATG without prior steroids. Clinical, demographic and laboratory data were collected and univariate analysis were performed.

Results 39 LT patients fulfilled inclusion/exclusion criteria. 13 did not achieve a 1-year LT-free survival and a further 5 did not achieve a 5-year LT free survival. Age, female sex, LT for

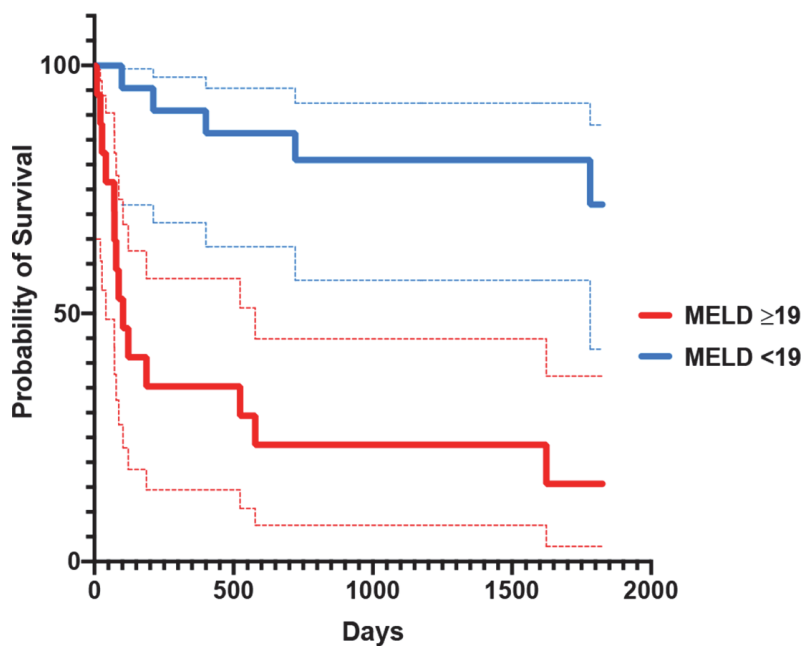

Abstract P084 Figure 1 an autoimmune liver disease, redo LT and pre-ATG AST, tacrolimus level, creatinine, albumin, haemoglobin, white cell count and differentials did not predict 1-year LT free survival. Variables predicting reduced 1-year LT free survival were:increased time from LT when receiving ATG (AUC 0.82, 95\% CI 0.69-0.96), histological overlap with chronic rejection (AUC 0.75 , 95\% CI 0.57-0.93), increased pre-ATG bilirubin (AUC 0.80, 95\% CI 0.66-0.94), INR (AUC 0.71, 95\%CI $0.52-0.89$ ) and MELD (AUC 0.86, 95\% CI 0.75-0.97). Survival analysis was performed to evaluate risk factors affecting 5-year LT free survival. Cut-off values for pre-ATG MELD and days post-LT receiving ATG were determined by Youden's Index. Histological overlap with chronic rejection (HR 3.33, 95\% CI 1.10-10.11), receiving ATG $>178$ days post-LT (HR 5.86, 95\% CI 1.82-18.83) and a pre-ATG MELD $\geq 19$ (HR 8.84, 95\% CI 3.25-24.04) (see figure 1) predicted a reduced 5 -year survival.

Conclusion ATG use in patients with steroid resistant $\mathrm{T}$ cellmediated rejection with a MELD $\geq 19$ is associated with suboptimal 5-year LT-free survival. ATG use and re-transplant candidacy needs to be re-evaluated in this clinical cohort.

\section{P085 VIRTUAL TRANSPLANT ASSESSMENT IS FEASIBLE AND MAY INCREASE ACCESS TO LIVER TRANSPLANTATION}

${ }^{1}$ Agimol Pradeep*, ${ }^{1}$ Faye Barker, ${ }^{2}$ Katie Ramos, ${ }^{1}$ Wendy Littlejohn, ${ }^{1}$ Oliver Tavabie, ${ }^{1}$ Chris Nicholson, ${ }^{1}$ Krishna Menon, ${ }^{2}$ Matthew Cramp, ${ }^{3}$ Neil McDougall, ${ }^{3} J o h n n y$ Cash, ${ }^{1}$ Varuna Aluvihare. ${ }^{1}$ King's College Hospital, UK; ${ }^{2}$ Plymouth Hospitals NHS Trust, UK; ${ }^{3}$ Royal Victoria Hospital, UK

\subsection{6/gutjnl-2021-BASL.93}

Background It has been demonstrated that patients living further from a transplant centre are less likely to be transplanted in the UK and abroad. King's College Hospital (KCH) has collaborated with teams in Plymouth, North Bristol and Belfast to establish satellite transplant centres (SLTCs) to address this. However, the COVID-19 pandemic threatened to exacerbate this healthcare inequity by reducing travel and stopping patients undergoing transplant assessment at $\mathrm{KCH}$. In response to this, we developed a virtual transplant assessment (VTA) pathway to ensure that patients at SLTCs were not disadvantaged through the pandemic.

Methods Data were retrospectively collected from all patients referred from our SLTCs and discussed at $\mathrm{KCH}$ transplant listing meetings between April 2020 and April 2021. Patients were either assessed face-to-face or virtually depending on the 'discussion in principle' (DIP) MDM between $\mathrm{KCH}$ and the corresponding SLTC. Demographic (age and sex), clinical (disease aetiology, smoking history, time of referral, time of full discussion and whether the patient was listed for transplant or transplanted) and laboratory data for calculation of prognostic scores were collected from the clinical notes. Continuous variables were analysed for normality using the D'Agostino and Pearson test and patients undergoing face-to-face assessments were directly compared with those undergoing VTA by $t$ test if normally distributed or Mann-Whitney $U$ test if non-normally distributed. Categorical data were analysed using Fisher's exact test. Time from listing to transplantation was compared between both groups by survival analysis.

Results During this time, 19 patients underwent VTA and 30 patients underwent face-to-face assessment. No patients were fully assessed from SLTCs between April and July 2020. 